\title{
Concordancia diagnóstica del puntaje de G leason en biopsia por punción y prostatectomía radical y sus consecuencias clínicas
}

\author{
Leonardo Arellano $\mathrm{H}^{1}, \mathrm{O}$ ctavio Castillo $\mathrm{C}^{2}$, \\ Esteban Metrebián $\mathrm{B}^{3}$.
}
Concordance of G leason histological scoring for prostatic cancer in needle biopsies and the surgical piece obtained during radical prostatectomy

Background: The Gleason histological score is an independent prognostic factor in prostate cancer that helps in therapeutic decisions. Aim: To analyze the concordance between the Gleason score of needle prostatic biopsy and the score from the study of the surgical piece obtained during radical prostatectomy. Material and methods: Retrospective analysis of 212 cases of prostate cancer, diagnosed between February 1993 and March 2003. All had the Gleason histological scores from needle prostatic biopsies and in the surgical piece obtained during radical prostatectomy. All pathological studies were done by the same observer. Results: There was an exact concordance of Gleason scores between needle biopsy and the surgical piece in $49 \%$ of cases and a concordance of \pm 1 unit in $89 \%$. The concordance improved in the second half of the study period. No cases with a Gleason score of less than 5 were detected since 1999 in the surgical piece. Conclusions: The concordance of Gleason score of needle prostatic biopsies and surgical pieces is good. This concordance increases with the experience of the pathologist (Rev Méd Chile 2004; 132: 971-8).

(Key Words: Biopsy, needle; Prostatectomy; Prostatic neoplasms; Urogenital surgical procedures)

Recibido el 17 de noviembre, 2003. Aceptado en versión corregida el 1 de julio, 2004.

Unidad de Urología, Clínica Santa María, Laboratorio de Histopatología, Clínica Avansalud.

${ }^{1}$ Laboratorio de Histopatología, Clínica Avansalud. ${ }^{2}$ Unidad de Urología, Clínica Santa María. ${ }^{3}$ Becado de Urología, Clínica Santa María.

Correspondencia a: Dr. Leonardo Arellano H. Clínica Avansalud Providencia. Av. Salvador 130, Providencia. Santiago. FonoFax: 2237319. E mail: leoare@vtr.net 
$\mathrm{S}_{\mathrm{s}}^{\mathrm{e}}$ ha demostrado que la determinación del grado histológico es un importante factor pronóstico independiente y su uso se acepta en pacientes con adenocarcinoma de próstata ${ }^{1-8}$. El sistema de Gleason es el método más utilizado para la obtención del grado histológico ya que tiene una buena correlación intraobservador e interobservador ${ }^{1,4-6}$. Gleason, en su trabajo original, observó una reproducibilidad exacta de 50\% y una puntuación de más menos una unidad en el 85\% de los $\operatorname{casos}^{5}$ al comparar grado de Gleason en la punción y la prostatectomía. Este sistema está basado en el grado de diferenciación glandular y en el patrón de crecimiento del tumor en relación al estroma prostático, considerando sólo los dos patrones histológicos más frecuentes ${ }^{1-4}$.

El puntaje de Gleason, junto al estadio clínico y los niveles séricos de antígeno prostático específico (PSA) son variables preoperatorias cruciales para predecir el estado anatomopatológico, y de esta forma ayudar a determinar las conductas terapéuticas, con el fin de otorgar el máximo beneficio para el paciente ${ }^{1,3,5-8}$.

El tejido obtenido mediante la biopsia por punción o bien mediante la resección transuretral de próstata sirve para el diagnóstico y la graduación histológica de los adenocarcinomas de próstata. Por ello, la precisión con la que estas muestras reflejan la apariencia histológica del tumor primario es de gran importancia clínica y terapéutica ${ }^{9,10}$.

El objetivo de este estudio es evaluar la exactitud que presenta el puntaje de Gleason de las biopsias por punción para predecir el puntaje de Gleason en la pieza de la prostatectomía radical, correlacionar ambos y ver la evolución del diagnóstico realizado por el anatomopatólogo de acuerdo a su experiencia en el tiempo transcurrido.

\section{Material y MÉTOdo}

Se analizaron en forma retrospectiva casos con diagnóstico de adenocarcinoma de próstata en biopsias por punción y piezas de prostatectomía radical realizadas en el período entre el 26 de febrero de 1993 y 15 de marzo de 2003. La totalidad del material de las biopsias y de las piezas de las prostatectomías fueron evaluadas por un solo anatomopatólogo (LAH).
Las biopsias por punción fueron tomadas con agujas de 18 gauge guiadas por ecografía transrectal, con un mínimo de 12 muestras por paciente, dos por cada sextante. Las piezas de las prostatectomías radicales se analizaron completamente en forma seriada mediante cortes de 3 milímetros de espesor desde el ápex hasta la base.

En todas las biopsias por punción se realizó tinción con hematoxilina-eosina y Van Gieson; en las biopsias de prostatectomía radical, tinción con hematoxilina-eosina y algunos casos Van Gieson.

Para el grado histológico se utilizó el sistema de Gleason. Este sistema tiene en cuenta las variantes histopatológicas del cáncer considerando cinco patrones histológicos siendo el patrón 1 el más diferenciado y el patrón 5 el más indiferenciado. Luego se suma el patrón primario, que es el predominante (más del 50\% de la muestra), con el patrón secundario, que es el segundo en importancia, en cuanto a porcentaje de la muestra y debe ser más de $5 \%$ (patrón secundario), de esta forma se determina el puntaje final del Gleason. $\mathrm{Si}$ existe un solo patrón, la suma se realiza considerándolo como patrón primario y secundario ${ }^{4}$.

Se comparó la biopsia por punción y la pieza de la prostatectomía radical de cada paciente, evaluando tanto el puntaje de Gleason final, como así también la suma del patrón primario y secundario en cada caso, determinando de esta forma la correlación existente entre ambos diagnósticos en cada caso.

Para comparar la correlación de la suma del patrón primario más el patrón secundario entre la biopsia y la prostatectomía, sólo se consideraron aquellos pacientes que lo tenían al momento del diagnóstico anatomopatológico.

Para un mejor análisis se reagruparon los puntajes de Gleason en 4 grupos de riesgo: puntaje de Gleason 2-4 (grupo bien diferenciado), puntaje de Gleason 5-6 (grupo moderadamente diferenciado), puntaje de Gleason 7 (grupo intermedio) y puntaje de Gleason 8-10 (grupo poco diferenciado). De esta forma, se determinó la correlación exacta entre los grupos y el número de pacientes, en los cuales se presentaba un cambio en el grupo, luego de comparar los puntajes de Gleason entre la biopsia y la prostatectomía.

Para analizar la evolución en el tiempo de la capacidad para predecir el puntaje de Gleason de la prostatectomía, se dividieron los casos en dos 
mitades iguales. En la primera mitad se incluyeron los pacientes del 26 de febrero de 1993 al 7 de septiembre de 1999, y en la segunda mitad los pacientes del 21 de septiembre de 1999 al 15 de marzo de 2003. Se evaluaron en forma separada los datos obtenidos en cada grupo y posteriormente se compararon los resultados de las mitades.

\section{RESULTADOs}

Se recolectaron 212 casos, la distribución global de los puntajes de Gleason de la biopsia por punción y de la pieza de la prostatectomía radical se encuentran detalladas en la Tabla 1. En la misma se puede observar que a pesar que 12 pacientes fueron clasificados como bien diferenciados en la biopsia por punción (Gleason 2-4), sólo uno de ellos fue bien diferenciado al examinar la pieza de la prostatectomía radical. La mayoría de los casos presentaron puntaje de Gleason entre 5 y 7 tanto para la biopsia por punción $(87,7 \%$ de los casos) como para la prostatectomía (90,6\% de los casos).

La Tabla 2 muestra la correlación entre el puntaje de Gleason en la biopsia por punción y en la pieza de la prostatectomía radical. Se observa que existió 49,5\% de correlación exacta entre ambas muestras, y en $88,7 \%$ de los casos se obtuvo una concordancia de \pm 1 unidad entre el puntaje de la biopsia por punción y de la prostatectomía radical. Hubo $42 \%$ de subgraduación y 8,5\% de sobregraduación con respecto al puntaje de la prostatectomía radical en general.

Sólo 181 pacientes tenían el diagnóstico del patrón primario y secundario en la biopsia por

Tabla 1. D istribución del puntaje de G leason en las biopsias por punción y en la prostatectomía radical

\begin{tabular}{|ccccc|}
\hline Puntaje de Gleason & \multicolumn{4}{c|}{ Número de pacientes } \\
& Biopsia por punción & \multicolumn{2}{c|}{ Prostatectomía radical } \\
& $\mathrm{n}$ & $\%$ & $\mathrm{n}$ & $\%$ \\
\hline $2-4$ & 12 & 5,7 & 1 & 0,5 \\
$5-6$ & 125 & 58,9 & 91 & 42,9 \\
7 & 61 & 28,8 & 101 & 47,6 \\
$8-10$ & 14 & 6,6 & 19 & 9 \\
Total & 212 & 100 & 212 & 100 \\
\hline
\end{tabular}

Tabla 2. Correlación del puntaje de G leason entre la biopsia por punción y la pieza de la prostatectomía radical

\begin{tabular}{|c|c|c|c|c|c|c|c|c|}
\hline \multirow{2}{*}{$\begin{array}{l}\text { Puntaje de Gleason en } \\
\text { la biopsia por punción }\end{array}$} & \multicolumn{8}{|c|}{ Puntaje de Gleason en la pieza de la prostatectomía radical } \\
\hline & 3 & 4 & 5 & 6 & 7 & 8 & 9 & Total \\
\hline 3 & & & 2 & 3 & 1 & & & 6 \\
\hline 4 & & 1 & 2 & 3 & & & & 6 \\
\hline 5 & & & 12 & 21 & 10 & 1 & & 44 \\
\hline 6 & & & 8 & 35 & 35 & 2 & 1 & 81 \\
\hline 7 & & & 1 & 4 & 50 & 6 & & 61 \\
\hline 8 & & & & & 5 & 5 & 2 & 12 \\
\hline 9 & & & & & & & 2 & 2 \\
\hline Total & & 1 & 25 & 66 & 101 & 14 & 5 & 212 \\
\hline
\end{tabular}


punción y en la pieza de la prostatectomía radical, su concordancia se muestra en la Tabla 3. En $39,2 \%$ de los casos existió una correlación exacta entre los patrones primario y secundario de ambos grupos.

En la Tabla 4 se detalla la división del puntaje de Gleason en los cuatro grupos de riesgo, demostrándose una correlación exacta entre los grupos en $64,1 \%$ de los casos, mientras que en $35,8 \%$ de las muestras analizadas existió un cambio a un grupo diferente, especialmente en los grupos bien y moderadamente diferenciados (2-4 y 5-6) que pasaron a un grupo más alto en la prostatectomía. Se evidenció un ascenso de grupo de riesgo en $31,1 \%$ y un descenso de grupo en $4,7 \%$.

Tabla 3. Concordancia del patrón primario y secundario del puntaje de G leason en la biopsia por punción y en la prostatectomía radical

\begin{tabular}{|c|c|c|c|c|c|c|c|c|c|c|c|c|c|}
\hline \multirow{2}{*}{$\begin{array}{l}\text { Puntaje de } \\
\text { Gleason } \\
\text { en la BP } \\
\left(\mathrm{P} 1^{\text {nio }}+P 2^{\text {nio }}\right)\end{array}$} & \multirow[b]{2}{*}{$2+3$} & \multirow[b]{2}{*}{$2+4$} & \multirow[b]{2}{*}{$2+5$} & \multirow[b]{2}{*}{$3+2$} & \multicolumn{5}{|c|}{$\begin{array}{l}\text { Puntaje de Gleason en la PR } \\
\left(\mathrm{P}^{1 \text { io }}+\mathrm{P}^{\text {rio }}\right)\end{array}$} & \multirow[b]{2}{*}{$4+5$} & \multirow[b]{2}{*}{$5+3$} & \multirow[b]{2}{*}{$5+4$} & \multirow[b]{2}{*}{ Total } \\
\hline & & & & & $3+3$ & $3+4$ & $3+5$ & $4+3$ & $4+4$ & & & & \\
\hline $2+1$ & & & & 1 & 1 & & & & & & & & 2 \\
\hline $2+2$ & 1 & & & 1 & & & & & & & & & 2 \\
\hline $2+3$ & & 1 & & 8 & 10 & 1 & & & & & & & 20 \\
\hline $2+4$ & & & & & 1 & & & & & & & & 1 \\
\hline $2+5$ & & & & & & & & 1 & & & & & 1 \\
\hline $3+2$ & & & & 3 & 8 & 6 & & 1 & & & & & 18 \\
\hline $3+3$ & & & & 8 & 30 & 23 & 1 & 8 & & & & & 70 \\
\hline $3+4$ & & & & 1 & 1 & 25 & & 9 & 2 & & & & 38 \\
\hline $3+5$ & & & & & & 2 & 1 & & & & 1 & & 4 \\
\hline $4+3$ & & & & & 2 & 5 & 1 & 9 & 1 & & & & 18 \\
\hline $4+4$ & & & & & & & & 2 & & & & & 2 \\
\hline $4+5$ & & & & & & & & & & 1 & & & 1 \\
\hline $5+3$ & & & & & & & & & & 1 & 1 & 1 & 3 \\
\hline $5+4$ & & & & & & & & & & & & 1 & 1 \\
\hline Total & 1 & 1 & & 22 & 53 & 62 & 3 & 30 & 3 & 2 & 2 & 2 & 181 \\
\hline
\end{tabular}

BP: Biopsia por punción; PR: Prostatectomía radical; P1rio: Patrón primario; P2rio: Patrón secundario.

Tabla 4. Correlación entre los grupos según el puntaje de G leason en la biopsia por punción y en la prostatectomía radical

\begin{tabular}{|cccccc|}
\hline $\begin{array}{c}\text { Grupos según el } \\
\text { puntaje de } \\
\text { Gleason en la BP }\end{array}$ & $2-4$ & $5-6$ & 7 & $8-10$ & Total \\
\hline $2-4$ & 1 & 10 & 1 & & 12 \\
$5-6$ & & 5 & 45 & 4 & 125 \\
7 & & 50 & 5 & 9 & 61 \\
$8-10$ & 1 & 91 & 101 & 19 & 212 \\
Total & & & \\
\hline
\end{tabular}

BP: Biopsia por punción; PR: Prostatectomía radical. 
En las Tablas 5 y 6 se observa que entre los primeros 106 pacientes, existieron 45 casos con puntaje de Gleason 3, 4 y 5 en las biopsias por punción, mientras que entre los segundos 106 pacientes sólo se diagnosticaron en la biopsia 11 pacientes con puntaje de Gleason 5 y no hubo pacientes con Gleason 3 ni 4. En la prostatectomía no existe en los últimos años casos de puntaje de Gleason menor de 5.

En la Tabla 7 se resume la correlación del puntaje de Gleason en la biopsia por punción y en la prostatectomía radical según su evolución en el tiempo. Se puede observar que en los primeros 106 pacientes existió una correlación \pm 1 unidad del score de Gleason en el 83\% de los casos, mientras que en los segundos 106 pacientes la correlación fue del 94,3\%. También hay que destacar que en el primer grupo existió una subgraduación en el $54,7 \%$ de los pacientes a diferencia del segundo grupo en donde la subgraduación ocurrió en $29,2 \%$ de los casos.

Tabla 5. Distribución y concordancia del puntaje de G leason entre el 26 de febrero de 1993 hasta el 7 de septiembre de 1999

\begin{tabular}{|c|c|c|c|c|c|c|c|c|}
\hline \multirow{2}{*}{$\begin{array}{l}\text { Puntaje de Gleason } \\
\text { en la BP }\end{array}$} & \multicolumn{8}{|c|}{$\begin{array}{c}\text { Pacientes 1-106 } \\
\text { Puntaie de Gleason en la nieza de la PR }\end{array}$} \\
\hline & 3 & 4 & 5 & 6 & 7 & 8 & 9 & Total \\
\hline 3 & & & 2 & 3 & 1 & & & 6 \\
\hline 4 & & 1 & 2 & 3 & & & & 6 \\
\hline 5 & & & 7 & 20 & 5 & 1 & & 33 \\
\hline 6 & & & 2 & 17 & 15 & 2 & 1 & 37 \\
\hline 7 & & & & 3 & 11 & 3 & & 17 \\
\hline 8 & & & & & 2 & 4 & & 6 \\
\hline 9 & & & & & & & 1 & 1 \\
\hline Total & & 1 & 13 & 46 & 34 & 10 & 2 & 106 \\
\hline
\end{tabular}

BP: Biopsia por punción; PR: Prostatectomía radical.

Tabla 6. D istribución y concordancia del puntaje de G leason entre el 21 de septiembre de 1999 al 15 de marzo de 2003

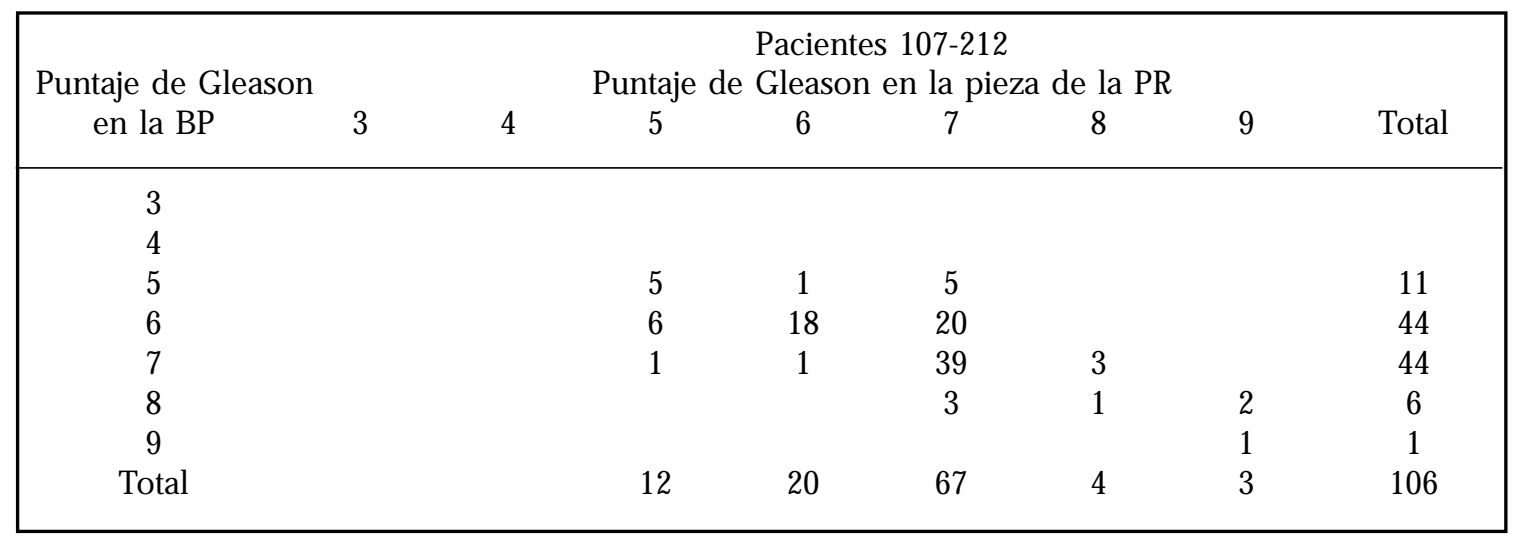

BP: Biopsia por punción; PR: Prostatectomía radical. 
Tabla 7. Precisión del puntaje de G leason de la biopsia por punción para predecir el puntaje de G leason de la prostatectomía radical y su evolución en el tiempo

\begin{tabular}{|c|c|c|c|c|c|c|c|c|c|c|}
\hline \multirow[t]{2}{*}{ Grupo } & \multicolumn{2}{|c|}{$\begin{array}{l}\text { Correlación } \\
\text { Exacta }\end{array}$} & \multicolumn{2}{|c|}{$\begin{array}{l}\text { Diferencia } \\
\text { por una } \\
\text { Unidad }\end{array}$} & \multicolumn{2}{|c|}{$\begin{array}{l}\text { Diferencia } \\
\text { por } 2 \text { o más } \\
\text { Unidades }\end{array}$} & \multicolumn{2}{|c|}{ Subgraduación } & \multicolumn{2}{|c|}{ Sobregraduación } \\
\hline & $\mathrm{n}$ & $\%$ & $\mathrm{n}$ & $\%$ & $\mathrm{n}$ & $\%$ & $\mathrm{n}$ & $\%$ & $\mathrm{n}$ & $\%$ \\
\hline $1-106$ & 41 & 38,7 & 47 & 44,3 & 18 & 17 & 58 & 54,72 & 7 & 6,6 \\
\hline $107-212$ & 64 & 60,4 & 36 & 34 & 6 & 5,7 & 31 & 29,24 & 11 & 10,4 \\
\hline Total & 105 & 49,5 & 83 & 39,1 & 24 & 11,3 & 89 & 42 & 18 & 8,5 \\
\hline
\end{tabular}

DisCUSIÓN

El grado histológico es uno de los más importantes predictores independientes del comportamiento biológico del adenocarcinoma de próstata. La gravedad del cáncer aumenta a medida que las células malignas recién formadas pierden su diferenciación. Debido a ello, el puntaje de Gleason presenta un importante rol pronóstico ya que permite predecir el estadio patológico, teniendo en cuenta que tiene correlación con los índices de penetración capsular, positividad de los márgenes, invasión de vesículas seminales y compromiso de ganglios linfáticos. En consecuencia, la graduación precisa del adenocarcinoma prostático es un componente fundamental en la planificación de las diferentes opciones terapéuticas ${ }^{4}$.
Nuestros resultados demuestran que existe una buena correlación entre el puntaje de Gleason de la biopsia por punción y el de la pieza de prostatectomía radical ya que $49,5 \%$ de los casos presentó una correlación exacta entre ambos valores y en $88,7 \%$ de los casos se obtuvo una concordancia de \pm 1 unidad entre el puntaje de la biopsia por punción y de la prostatectomía radical.

Estos hallazgos concuerdan con otros estudios de similares características. En Tabla 8 se observa que la correlación exacta entre el puntaje de Gleason de la biopsia por punción y el de la pieza de la prostatectomía radical se encuentra en un rango entre 30-59\%. Las biopsias por punción subgradúan el grado histológico del tumor en 33$45 \%$ de los casos y lo sobregradúan en 4-32\% ${ }^{9,11-15}$.

Tabla 8. Correlación entre la graduación histológica de la biopsia por punción y la prostatectomía radical

\begin{tabular}{|lcccc|}
\hline Serie & No Casos & $\begin{array}{c}\text { Correlación } \\
\text { exacta (\%) }\end{array}$ & $\begin{array}{c}\text { Diferencia por una } \\
\text { Unidad (\%) }\end{array}$ & $\begin{array}{c}\text { Diferencia por 2 } \\
\text { o más Unidades }\end{array}$ \\
\hline Catalona et al & & 59 & No disponible & No disponible \\
Garnet et al1 $^{11}$ & 66 & 30 & 42 & 28 \\
Mills et al $^{13}$ & 115 & 51 & 23 & 26 \\
Spires et all & 53 & 58 & 36 & 6 \\
Bostwick $^{15}$ & 67 & 35 & 39 & 26 \\
Cookson et al $^{9}$ & 316 & 31 & 43 & 11 \\
Serie actual & 226 & 50 & 39 & \\
\hline
\end{tabular}


Los errores de graduación probablemente se deban a errores en la toma de muestras, a biopsias con pequeños volúmenes tumorales y a la heterogeneidad tumoral $1,2,4,5$. Bostwick et al compararon el puntaje de Gleason de la biopsia por punción con el de la prostatectomía en 316 pacientes, informando una correlación exacta en $35 \%$ de los casos, en sólo 39\% existía una diferencia de una unidad del puntaje de Gleason. En su estudio no encontró relación entre el error en la graduación de la biopsia por punción con el volumen de adenocarcinoma en las muestras ${ }^{15}$.

Cookson et al describieron una concordancia exacta de $31 \%$ entre el puntaje de Gleason de la biopsia y el de la prostatectomía de 226 pacientes. Señalando que en $26 \%$ hubo una discrepancia de dos o más unidades del puntaje. Determinó además, que $54 \%$ de las biopsias fueron subgraduadas y $15 \%$ sobregraduadas ${ }^{9}$.

$\mathrm{Al}$ comparar los cuatro grupos de riesgo (grupos 2, 3, 4; grupos 5, 6; grupo 7 y grupos 8, 9, 10) existe una concordancia exacta de $64,1 \%$ entre los grupos determinados en la biopsia por punción y los de la pieza de la prostatectomía radical y una discordancia de 35,8\% produciendo un cambio a un grupo diferente en la pieza de prostatectomía. El significado clínico de estas discrepancias puede ser relevante y debiera tenerse en cuenta al momento de tomar las decisiones terapéuticas, especialmente en el grupo intermedio (Gleason 7), ya que un cambio en el grupo puede significar también un cambio en las decisiones terapéuticas. Cookson et al reportaron en su estudio que $54 \%$ de los pacientes presentaron un cambio a un grupo diferente. Determinaron, además, que el valor predictivo positivo de la

\section{REFERENCIAS}

1. Bostwick DG, Eble JN. Urologic surgical pathology. En: Neoplasms of the prostate. Missouri, Mosby, 1997; 343-422.

2. EPSTein JI, PotTeR SR. The pathological interpretation and significance of prostate needle biopsy findings: implications and current controversies. J Urol 2001; 166: 402-10.

3. Bostwick DG, Grignon DJ, Hammond EH, Amin MB, Cohen M, Crawford D, Gospadarowicz M. Prognos- biopsia por punción para el Gleason 7 es de sólo $55 \%{ }^{9}$.

Dividimos nuestro estudio en dos partes para analizar la precisión del diagnóstico del grado histológico de la biopsia por punción a través del tiempo. Observamos un incremento considerable en la precisión para determinar el puntaje de Gleason en la biopsia por punción a medida que el anatomopatólogo adquiere más experiencia, ya que es capaz de diagnosticar mejor el grado histológico de los adenocarcinomas de próstata, aun con pequeños volúmenes de tejido en las biopsias por punción ${ }^{2,4}$. Epstein et al demostraron que residentes de patología e incluso anatomopatólogos con experiencia mostraron mejoras significativas en la graduación histológica de los adenocarcinomas prostáticos, luego de un programa educacional dirigido ${ }^{2}$. Esto además refuerza la idea de que es necesario una dedicación y una curva de aprendizaje en el uso de la graduación histológica de Gleason, como lo han descrito otros autores, ya que pueden existir cambios en la perspectiva e interpretación de los patrones histológicos de Gleason 2,4,16,17.

La no existencia de puntaje bien diferenciado ${ }^{2-4}$ en las prostatectomías, especialmente en los últimos años, pone una alerta a aquellas biopsias diagnosticadas por punción y que tienen Gleason menor que 5 .

Un ejemplo aparece en una revisión de Epstein et al en el año 2001, en donde proponen que no se debe asignar los puntajes de Gleason 2, 3 y 4 a los adenocarcinomas de próstata diagnosticados en la biopsia por punción, debido a que por lo general los adenocarcinomas bien diferenciados están ubicados en la zona de transición y por lo general presentan pequeños volúmenes tumorales $2,16,17$.

tic factors in prostate cancer: College of American Pathologist consensus statement 1999. Arch Pathol Lab Med 2000; 124: 995-1000.

4. EPSTEIN JI. Prostate biopsy interpretation. Second edition. En: Grading of prostatic adenocarcinomas. Philadelphia, Lippincott \& Raven, 1995; 65132.

5. Bostwick DG, IczKowski KA. Interpretación de la biopsia de próstata: Conceptos actuales, 1999. Clínicas de Urología de Norteamérica 1999; 3: 461-79. 
6. Bostwick DG. Grading prostate cancer. Am J Clin Pathol 1994; 102: S38-56.

7. Partin aW, Kattan MW, Subong EN, Walsh PC, Wojno KJ, Oesterling JE, Scardino PT. Combination of prostate-specific antigen, clinical stage, and Gleason score to predict pathological stage of localized prostate cancer: A multi-institutional update. JAMA 1997; 277: 1445-51.

8. Возтwick DG. Evaluating Prostate Needle Biopsy: Therapeutic and Prognostic Importance. CA Cancer J Clin 1997; 47: 297-319.

9. CoOkson MS, Fleshner NE, Soloway SM, Fair WR. Correlation between Gleason score of needle biopsy and radical prostatectomy specimen: Accuracy and clinical implications. J Urol 1997; 157: $559-62$.

10. San Francisco IF, DeWolf WC, Rosen S, Upton M, OLumi AF. Extended prostate needle biopsy improves concordance of Gleason grading between prostate needle biopsy and radical prostatectomy. J Urol 2003; 169: 136-40.

11. Catalona WJ, Stein AJ, Fair WR. Grading errors in prostatic needle biopsies: relation to the accuracy of tumor grade in predicting pelvic lymph node metastases. J Urol 1982; 127: 919-22.
12. Garnett JE, Oyasu R, Grayhack JT. The accuracy of diagnostic biopsy specimens in predicting tumor grades by Gleason's classification of radical prostatectomy specimens. J Urol 1984; 131: 690-3.

13. MILS SE, FowLER JE JR. Gleason histologic grading of prostatic carcinoma. Correlations between biopsy and prostatectomy specimens. Cancer 1986; 57: 346-9.

14. Spires SE, Cibull ML, Wood DP JR, Miler S, Spires $\mathrm{SM}$, BANKS ER. Gleason histologic grading in prostatic carcinoma. Correlation of 18 gauge core biopsy with prostatectomy. Arch Pathol Lab Med 1994; 118: 705-8.

15. BоsтwICK DG. Gleason grading of prostatic needle biopsies. Comelation with grade in 316 matched prostatectomies. Am J Surg Pathol 1994; 18: 796-803.

16. Smith EB, Frierson HF, Mils SE, Boyd JC, TheodoRESCU DT. Gleason scores of prostate biopsy and radical prostatectomy specimens over the past 10 years: Is there evidence for systematic upgrading? Cancer 2002; 94: 2282-7.

17. Gimiand FD, Gleason DF, Hunt WC, Stone N, HaRlan LC, Key CR. Trends in Gleason score for prostate cancer diagnosed between 1983 and 1993. J Urol 2001; 165: 846-50. 